UDC 517.9

R. P. Agarwal (Florida Inst. Technol., USA),

J. V. Manojlović* (Univ. Niš, Serbia)

\title{
ASYMPTOTIC BEHAVIOR \\ OF POSITIVE SOLUTIONS OF FOURTH-ORDER \\ NONLINEAR DIFFERENCE EQUATIONS
}

\section{АСИМПТОТИЧНА ПОВЕДІНКА ДОДАТНИХ РОЗВ'ЯЗКІВ НЕЛІНІЙНИХ РІЗНИЦЕВИХ РІВНЯНЬ ЧЕТВЕРТОГО ПОРЯДКУ}

We consider a class of fourth-order nonlinear difference equations of the form

$$
\Delta^{2}\left(p_{n}\left(\Delta^{2} y_{n}\right)^{\alpha}\right)+q_{n} y_{n+3}^{\beta}=0, \quad n \in \mathbb{N},
$$

where $\alpha, \beta$ are the ratios of odd positive integers, and $\left\{p_{n}\right\},\left\{q_{n}\right\}$ are positive real sequences defined for all $n \in \mathbb{N}\left(n_{0}\right)$. We establish necessary and sufficient conditions for the existence of nonoscillatory solutions with specific asymptotic behavior under suitable combinations of the convergence or divergence conditions of the sums

$$
\sum_{n=n_{0}}^{\infty} \frac{n}{p_{n}^{1 / \alpha}} \text { and } \sum_{n=n_{0}}^{\infty}\left(\frac{n}{p_{n}}\right)^{1 / \alpha} .
$$

Розглянуто клас нелінійних різницевих рівнянь четвертого порядку, що мають вигляд

$$
\Delta^{2}\left(p_{n}\left(\Delta^{2} y_{n}\right)^{\alpha}\right)+q_{n} y_{n+3}^{\beta}=0, \quad n \in \mathbb{N},
$$

де $\alpha, \beta$ с співвідношеннями непарних додатних цілих чисел, а $\left\{p_{n}\right\},\left\{q_{n}\right\}-$ додатними дійсними послідовностями, визначеними для всіх $n \in \mathbb{N}\left(n_{0}\right)$. Встановлено необхідні і достатні умови існування неколивних розв'язків із специфічною асимптотичною поведінкою у випадку прийнятних комбінацій умов збіжності або розбіжності сум

$$
\sum_{n=n_{0}}^{\infty} \frac{n}{p_{n}^{1 / \alpha}} \quad \text { та } \quad \sum_{n=n_{0}}^{\infty}\left(\frac{n}{p_{n}}\right)^{1 / \alpha}
$$

1. Introduction. In the last few years, there has been an increasing interest in the study of oscillatory and asymptotic behavior of solutions of difference equations (see monographs $[1,2]$ and the references therein). Compared to second-order difference equations, the study of higher-order equations (see [3-8]) and, in particular, fourthorder difference equations (see [9-14]) has received considerably less attention.

In this paper we are concerned with the fourth-order quasilinear difference equation

$$
\Delta^{2}\left(p_{n}\left(\Delta^{2} y_{n}\right)^{\alpha}\right)+q_{n} y_{n+3}^{\beta}=0, \quad n \in \mathbb{N}\left(n_{0}\right),
$$

${ }^{*}$ Supported by Ministry of Science of Republic of Serbia (Grant 144003). 
where $\mathbb{N}\left(n_{0}\right)=\left\{n_{0}, n_{0}+1, n_{0}+2, \ldots\right\}, n_{0}$ is a positive integer, $\Delta$ is the forward difference operator defined by $\Delta y_{n}=y_{n+1}-y_{n}, \alpha$ and $\beta$ are ratios of odd positive integers and $\left\{p_{n}\right\}$ and $\left\{q_{n}\right\}$ are positive real sequence defined for all $n \in \mathbb{N}\left(n_{0}\right)$.

By a solution of (1.1) we mean a real sequence $\left\{y_{n}\right\}$ satisfying (1.1) for $n \in \mathbb{N}\left(n_{0}\right)$. A nontrivial solution $\left\{y_{n}\right\}$ of equation (1.1) is called oscillatory if for every $M \in \mathbb{N}$ there exist $m, n \in \mathbb{N}, M \leq m<n$ such that $x_{m} x_{n}<0$, otherwise, it is nonoscillatory. Equation (1.1) is called oscillatory if all its solutions are oscillatory.

Oscillatory and nonoscillatory behavior of solutions of (1.1) under the condition

$$
\sum_{n=n_{0}}^{\infty} \frac{n}{p_{n}^{1 / \alpha}}=\infty \quad \text { and } \quad \sum_{n=n_{0}}^{\infty}\left(\frac{n}{p_{n}}\right)^{1 / \alpha}=\infty
$$

have been considered by Thandapani and Selvaraj in [13] and Agarwal and Manojlović in [15]. The aim of this paper is to proceed further in this direction and to obtain a more detailed information on the asymptotic behavior of nonoscillatory solutions of (1.1), under the assumptions which was not yet considered. Namely, we will investigate the structure of the set of positive solutions of (1.1) under each of the following conditions:

$$
\begin{array}{ll}
\sum_{n=n_{0}}^{\infty} \frac{n}{p_{n}^{1 / \alpha}}<\infty \quad \text { and } \quad \sum_{n=n_{0}}^{\infty}\left(\frac{n}{p_{n}}\right)^{1 / \alpha}=\infty, \\
\sum_{n=n_{0}}^{\infty} \frac{n}{p_{n}^{1 / \alpha}}=\infty \quad \text { and } \quad \sum_{n=n_{0}}^{\infty}\left(\frac{n}{p_{n}}\right)^{1 / \alpha}<\infty, \\
\sum_{n=n_{0}}^{\infty} \frac{n}{p_{n}^{1 / \alpha}}<\infty \quad \text { and } \quad \sum_{n=n_{0}}^{\infty}\left(\frac{n}{p_{n}}\right)^{1 / \alpha}<\infty .
\end{array}
$$

We emphasize that if (1.3) holds, then $\alpha<1$ and if (1.4) holds, then $\alpha \geq 1$.

Under assumptions (1.2)-(1.5), the following four sequences play a special role in the set of positive solutions of (1.1):

$$
\begin{array}{ll}
\alpha_{n}=1, & \gamma_{n}=\sum_{s=n_{0}}^{n-1}(n-s-1)\left(\frac{s}{p_{s}}\right)^{\frac{1}{\alpha}}, \\
\beta_{n}=n, & \delta_{n}=\sum_{s=n}^{\infty}(s-n+1)\left(\frac{1}{p_{s}}\right)^{\frac{1}{\alpha}} .
\end{array}
$$

Under the condition (1.2), Thandapani, Selvaraj in [13] established necessary and sufficient conditions for the existence of positive solutions of the following two types:

$$
\begin{array}{ll}
y_{n} \sim c \alpha_{n} \quad \text { as } \quad n \rightarrow \infty, & 0<c<\infty, \\
y_{n} \sim c \gamma_{n} \quad \text { as } \quad n \rightarrow \infty, & 0<c<\infty .
\end{array}
$$

Namely, they proved the following two theorems:

Theorem A. Suppose that (1.2) holds. A necessary and sufficient condition for the equation (1.1) to have a positive solution $\left\{y_{n}\right\}$ which satisfies (1.6) is that 


$$
\sum_{n=n_{0}}^{\infty} \frac{n}{p_{n}^{1 / \alpha}}\left(\sum_{s=n}^{\infty}(s-n) q_{s}\right)^{\frac{1}{\alpha}}<\infty
$$

Theorem B. Suppose that (1.2) holds. A necessary and sufficient condition for the equation (1.1) to have a positive solution $\left\{y_{n}\right\}$ which satisfies (1.7) is that

$$
\sum_{n=n_{0}}^{\infty} q_{n} \gamma_{n+3}^{\beta}<\infty
$$

Moreover, a solution $\left\{y_{n}\right\}$ satisfying (1.6) is minimal in the set of eventually positive solution of (1.1), while a solution $\left\{y_{n}\right\}$ satisfying (1.7) is maximal in the set of eventually positive solution of (1.1). Namely, there exists positive constants $k_{1}, k_{2}$ such that

$$
k_{1} \leq y_{n} \leq k_{2} \gamma_{n} \quad \text { for all large } n
$$

In this paper, we are going to investigate asymptotic behavior of positive solutions as $n \rightarrow \infty$ under the other three conditions (1.3)-(1.5). If (1.3) is satisfied, we give necessary and sufficient conditions for the existence of positive solutions satisfying (1.7) and

$$
y_{n} \sim c \delta_{n} \quad \text { as } \quad n \rightarrow \infty, \quad 0<c<\infty .
$$

It is observed that a solution satisfying (1.8) is "minimal" in the set of all eventually positive solution of (1.1), while a solution satisfying (1.7) is "maximal" in the set of all eventually positive solution of (1.1).

If (1.4) holds, in the set of all eventually positive solution of (1.1), a solution satisfying (1.6) may be a "minimal" solution, while a solution satisfying

$$
y_{n} \sim c \beta_{n} \quad \text { as } \quad n \rightarrow \infty, \quad 0<c<\infty,
$$

may be a "maximal" solution. We will establish necessary and sufficient conditions for the existence of this types of positive solutions.

If (1.5) holds, a solution $\left\{y_{n}\right\}$ of (1.1) having the asymptotic property (1.9) may be expected as a "minimal" solution in the set of all eventually positive solutions of (1.1). Moreover, a solution $\left\{y_{n}\right\}$ of (1.1) having the asymptotic property (1.8) is a "maximal" solution in the set of all eventually positive solutions of (1.1). Under the assumption (1.5), necessary and sufficient conditions are established for the existence of "minimal" and "maximal" positive solution.

2. Classification of positive solutions. We first have to classify the positive solutions in term of the signs of their differences, i.e., of the signs of

$$
\Delta y_{n}, \quad \Delta^{2} y_{n}, \quad \Delta\left(p_{n}\left(\Delta^{2} y_{n}\right)^{\alpha}\right)
$$

For a positive solution $\left\{y_{n}\right\}$ the next eight cases can occur: 


\begin{tabular}{c|c|c|c|c|c|c|c}
\hline Case & $\Delta\left(p_{n}\left(\Delta^{2} y_{n}\right)^{\alpha}\right)$ & $\Delta^{2} y_{n}$ & $\Delta y_{n}$ & Case & $\Delta\left(p_{n}\left(\Delta^{2} y_{n}\right)^{\alpha}\right)$ & $\Delta^{2} y_{n}$ & $\Delta y_{n}$ \\
\hline (a) & + & + & + & (e) & - & + & + \\
\hline (b) & + & + & - & (f) & - & + & - \\
\hline (c) & + & - & + & (g) & - & - & + \\
\hline (d) & + & - & - & (h) & - & - & - \\
\hline
\end{tabular}

The cases (d) and (h) never hold, because if $\Delta y_{n}<0$ and $\Delta^{2} y_{n}<0$ for all large $n$, we would have that $\lim _{n \rightarrow \infty} y_{n}=-\infty$, which contradicts the positivity of solution $\left\{y_{n}\right\}$. Similarly, if $\Delta\left(p_{n}\left(\Delta^{2} y_{n}\right)^{\alpha}\right)<0$, taking into account that from the equation $\Delta\left(p_{n}\left(\Delta^{2} y_{n}\right)^{\alpha}\right)$ is decreasing sequence, we would have that $\lim _{n \rightarrow \infty} p_{n}\left(\Delta^{2} y_{n}\right)^{\alpha}=$ $=-\infty$, that is $\Delta^{2} y_{n}<0$ for all large $n$, which eliminates cases (e) and (f).

Accordingly, for a positive solution $\left\{y_{n}\right\}$, the one of the following four cases holds:

$$
\begin{array}{llllll}
\text { (I) : } & \Delta\left(p_{n}\left(\Delta^{2} y_{n}\right)^{\alpha}\right)>0, & \Delta^{2} y_{n}>0, & \Delta y_{n}>0 & \text { for all large } & n, \\
\text { (II) : } & \Delta\left(p_{n}\left(\Delta^{2} y_{n}\right)^{\alpha}\right)>0, & \Delta^{2} y_{n}<0, & \Delta y_{n}>0 & \text { for all large } & n, \\
\text { (III) : } & \Delta\left(p_{n}\left(\Delta^{2} y_{n}\right)^{\alpha}\right)>0, & \Delta^{2} y_{n}>0, & \Delta y_{n}<0 & \text { for all large } & n, \\
\text { (IV) : } & \Delta\left(p_{n}\left(\Delta^{2} y_{n}\right)^{\alpha}\right)<0, & \Delta^{2} y_{n}<0, & \Delta y_{n}>0 & \text { for all large } & n .
\end{array}
$$

Moreover, we have the following two lemmas:

Lemma 2.1 (Lemma 2.1 [13]). Let $\left\{y_{n}\right\}$ be a positive solution of (1.1). If

$$
\sum_{n=n_{0}}^{\infty}\left(\frac{n}{p_{n}}\right)^{1 / \alpha}=\infty
$$

holds, then

$$
\Delta\left(p_{n}\left(\Delta^{2} y_{n}\right)^{\alpha}\right)>0 \text { for all large } n .
$$

Lemma 2.2. Let $\left\{y_{n}\right\}$ be a positive solution of (1.1) such that

$$
\Delta\left(p_{n}\left(\Delta^{2} y_{n}\right)^{\alpha}\right)>0, \quad \Delta^{2} y_{n}>0 \text { for all large } n .
$$

If

$$
\sum_{n=n_{0}}^{\infty} \frac{n}{p_{n}^{1 / \alpha}}=\infty
$$

holds, then $\Delta y_{n}>0$ for all large $n$.

Proof. From (2.1) we have

$$
p_{n}\left(\Delta^{2} y_{n}\right)^{\alpha} \geq p_{N}\left(\Delta^{2} y_{N}\right)^{\alpha}=c>0, \quad n \geq N,
$$

or 


$$
\Delta^{2} y_{n} \geq\left(\frac{c}{p_{n}}\right)^{1 / \alpha}, \quad n \geq N
$$

Multiplying by $n$ previous inequality and summing from $N$ to $n-1$, we obtain

$$
n \Delta y_{n}-N \Delta y_{N}-y_{n+1}+y_{N+1} \geq c^{1 / \alpha} \sum_{s=N}^{n-1} \frac{s}{p_{s}^{1 / \alpha}}, \quad n \geq N,
$$

which implies that

$$
n \Delta y_{n} \geq k+c^{1 / \alpha} \sum_{s=N}^{n-1} \frac{s}{p_{s}^{1 / \alpha}}, \quad n \geq N .
$$

Then, it follows from (2.2) that $n \Delta y_{n} \rightarrow \infty$ as $n \rightarrow \infty$ and consequently $\Delta y_{n}>0$ for all large $n$.

The lemma is proved.

Therefore, by all previous discussion we make the following conclusions:

Lemma 2.3. Let $\left\{y_{n}\right\}$ be a positive solution of (1.1).

(i) If (1.3) holds, then (I) or (II) or (III) holds;

(ii) If (1.4) holds, then (I) or (II) or (IV) holds;

(iii) If (1.5) holds, then (I) or (II) or (III) or (IV) holds.

3. Auxiliary lemmas. In this section we collect some lemmas which will be used in order to prove the main results. We will use the following fixed point theorem, which was proved in [14] and which can be considered as a discrete analog of Schauder's fixed point theorem.

Lemma 3.1. Suppose $X$ is a Banach space and $K$ is closed, bounded and convex subset of $X$. If $\mathcal{F}: K \rightarrow X$ is a continuous mapping such that $\mathcal{F}(K) \subset K$ and $\mathcal{F}(K)$ is uniformly Cauchy, then $\mathcal{F}$ has a fixed point in $K$.

Lemma 3.2. (i) If $\left\{\varphi_{n}\right\}$ is eventually negative sequence such that $\Delta \varphi_{n}>0$ and $\Delta^{2} \varphi_{n}<0$ for all large $n$, then $\lim _{n \rightarrow \infty} \Delta \varphi_{n}=0$.

(ii) If $\left\{\varphi_{n}\right\}$ is eventually positive sequence such that $\Delta \varphi_{n}<0$ and $\Delta^{2} \varphi_{n}>0$ for all large $n$, then $\lim _{n \rightarrow \infty} \Delta \varphi_{n}=0$.

Proof. (i) Since $\left\{\Delta \varphi_{n}\right\}$ is positive and decreasing sequence, there exists $\lim _{n \rightarrow \infty} \Delta \varphi_{n}=$ $=\varphi, 0 \leq \varphi<\infty$. If we suppose that $\varphi>0$, from $\Delta \varphi_{n} \geq \varphi$, we get

$$
\varphi_{n} \geq \varphi_{N}+\varphi(n-N), \quad n \geq N
$$

which obviously implies that $\lim _{n \rightarrow \infty} \varphi_{n}=\infty$, contradiction negativity of the sequence $\left\{\varphi_{n}\right\}$. Consequently, $\varphi=0$.

(ii) Since $\left\{\Delta \varphi_{n}\right\}$ is negative and increasing sequence, there exists $\lim _{n \rightarrow \infty} \Delta \varphi_{n}=\varphi$, $-\infty<\varphi \leq 0$. If we suppose that $\varphi<0$, from $\Delta \varphi_{n} \leq \varphi$, we get

$$
\varphi_{n} \leq \varphi_{N}+\varphi(n-N), \quad n \geq N
$$

which obviously implies that $\lim _{n \rightarrow \infty} \varphi_{n}=-\infty$, contradiction positivity of the sequence $\left\{\varphi_{n}\right\}$. Therefore, $\varphi=0$.

The lemma is proved. 
As a direct consequence of the previous lemma we have the following results for the (1.1).

Lemma 3.3. Let $\left\{y_{n}\right\}$ be a positive solution of (1.1).

(i) If a solution $\left\{y_{n}\right\}$ is of type (II), then $\lim _{n \rightarrow \infty} \Delta\left(p_{n}\left(\Delta^{2} y_{n}\right)^{\alpha}\right)=0$.

(ii) If a solution $\left\{y_{n}\right\}$ is of type (III), then $\lim _{n \rightarrow \infty} \Delta y_{n}=0$.

Next lemma gives some useful properties of positive solution of (1.1).

Lemma 3.4. Let $\left\{y_{n}\right\}$ be a positive solution of (1.1).

(i) If $\left\{y_{n}\right\}$ is of type (I), then $\lim _{n \rightarrow \infty} \frac{y_{n}}{n}>0$.

(ii) Let (1.3) or (1.5) holds and $\left\{y_{n}\right\}$ is a positive solution of (1.1) which satisfies (1.8). Then $\left\{y_{n}\right\}$ must be of type (III).

(iii) Let (1.4) holds and $\left\{y_{n}\right\}$ is a positive solution of (1.1) which satisfies (1.6). Then $\left\{y_{n}\right\}$ must be of type (II).

Proof. (i) Since $\left\{y_{n}\right\}$ is of type (I), there exists some $N \geq n_{0}$ such that (I) holds for all $n \geq N$. Then $\left\{\Delta y_{n}\right\}$ is the increasing sequence, so that $\Delta y_{n} \geq \Delta y_{n_{N}}>0$ for all $n \geq N$. Therefore, $y_{n} \geq y_{N}+\Delta y_{N}(n-N), n \geq N$, or

$$
\frac{y_{n}}{n} \geq \frac{y_{N}}{n}+\Delta y_{N}\left(1-\frac{N}{n}\right), \quad n \geq N .
$$

Accordingly, $\lim _{n \rightarrow \infty} \frac{y_{n}}{n} \geq \Delta y_{N}>0$.

(ii) Let $\left\{y_{n}\right\}$ be a positive solution of (1.1) which satisfies (1.8). If (1.5) holds, by Lemma 2.3, positive solution $\left\{y_{n}\right\}$ could be of type (I), (II), (III) or (IV). If we suppose that $\left\{y_{n}\right\}$ is of type (I), (II) or (IV), then $\lim _{n \rightarrow \infty} y_{n}=w_{0} \in(0, \infty]$. Moreover, (1.5) implies that $\lim _{n \rightarrow \infty} \delta_{n}=0$. But, then we would have that $\lim _{n \rightarrow \infty} \frac{y_{n}}{\delta_{n}}=\infty$, contradicting the assumption that $\left\{y_{n}\right\}$ satisfies (1.8). Therefore, the solution $\left\{y_{n}\right\}$ must be of type (III).

On the other hand, if (1.3) holds, by Lemma 2.3 positive solution $\left\{y_{n}\right\}$ is of type (I), (II) or (III) and therefore, using that (1.3) also implies that $\lim _{n \rightarrow \infty} \delta_{n}=0$, by the same arguments as in the previous case, we prove that $\left\{y_{n}\right\}$ is neither of type (I) nor (II), so it must be of type (III).

(iii) Let $\left\{y_{n}\right\}$ be a positive solution of (1.1) which satisfies (1.6). Then,

$$
\lim _{n \rightarrow \infty} \frac{y_{n}}{n}=0
$$

If (1.4) holds, by Lemma 2.3, positive solution $\left\{y_{n}\right\}$ is of type (I), (II), or (IV).

(a) If we suppose that $\left\{y_{n}\right\}$ is of type (I), then by (i) we have that $\lim _{n \rightarrow \infty} \frac{y_{n}}{n}>0$ contradicting (3.1).

(b) If we suppose that $\left\{y_{n}\right\}$ is of type (IV), then $\left\{p_{n}\left(\Delta^{2} y_{n}\right)^{\alpha}\right\}$ is decreasing, so that

$$
p_{n}\left(\Delta^{2} y_{n}\right)^{\alpha} \leq p_{N}\left(\Delta^{2} y_{N}\right)^{\alpha}=-K<0, \quad n \geq N
$$

Then, 


$$
n \Delta^{2} y_{n} \leq-K^{\frac{1}{\alpha}} \frac{n}{p_{n}^{1 / \alpha}}, \quad n \geq N
$$

and by summing obtained inequality from $N$ to $n-1$ we get

$$
\sum_{k=N}^{n-1} k \Delta^{2} y_{k} \leq-K^{\frac{1}{\alpha}} \sum_{k=N}^{n-1} \frac{k}{p_{k}^{1 / \alpha}}, \quad n \geq N
$$

or

$$
\sum_{k=N}^{n-1} k \Delta^{2} y_{k}=n \Delta y_{n}-N \Delta y_{N}-y_{n+1}+y_{N+1} \leq-K^{\frac{1}{\alpha}} \sum_{k=N}^{n-1} \frac{k}{p_{k}^{1 / \alpha}}, \quad n \geq N .
$$

Accordingly, taking into account that $\Delta y_{n}>0, n \geq N$, we have

$$
y_{n+1} \geq M+K^{\frac{1}{\alpha}} \sum_{k=N}^{n-1} \frac{k}{p_{k}^{1 / \alpha}}, \quad n \geq N
$$

where $M=y_{N+1}-N \Delta y_{N}$. Therefore, (1.4) implies that $\lim _{n \rightarrow \infty} y_{n}=\infty$, contradicting that $\left\{y_{n}\right\}$ satisfies (1.6).

Finally, the solution $\left\{y_{n}\right\}$ must be of type (II).

The lemma is proved.

We will also need the following lemma.

Lemma 3.5. Let $\left\{y_{n}\right\}$ be the positive solution of $(1.1)$ such that $\Delta\left(p_{n}\left(\Delta^{2} y_{n}\right)^{\alpha}\right)>$ $>0$ for all large $n$, then

$$
\lim _{n \rightarrow \infty} \frac{1}{n} \sum_{k=N}^{n-1} \sum_{j=k}^{\infty} q_{j} y_{j+3}^{\beta}=0 .
$$

Proof. Summing (1.1) from $N$ to $n-1$, we get

$$
\xi_{3}-\Delta\left(p_{n}\left(\Delta^{2} y_{n}\right)^{\alpha}\right)=\sum_{k=N}^{n-1} q_{k} y_{k+3}^{\beta}, \quad n \geq N+1,
$$

where $\xi_{3}=\Delta\left(p_{N}\left(\Delta^{2} y_{N}\right)^{\alpha}\right)$. Since $\left\{\Delta\left(p_{n}\left(\Delta^{2} y_{n}\right)^{\alpha}\right)\right\}$ is positive and decreasing sequence, it tends to a finite limit $w_{3} \geq 0$ as $n \rightarrow \infty$. Now, letting $n \rightarrow \infty$ in (3.3) we have that

$$
\sum_{k=N}^{\infty} q_{k} y_{k+3}^{\beta}<\infty
$$

Therefore, by Stolz's theorem we get

$$
\lim _{n \rightarrow \infty} \frac{1}{n} \sum_{k=N}^{n-1} \sum_{j=k}^{\infty} q_{j} y_{j+3}^{\beta}=\lim _{n \rightarrow \infty} \sum_{j=n}^{\infty} q_{j} y_{j+3}^{\beta}=0 .
$$

The lemma is proved.

4. "Maximal" and "minimal" positive solutions of (1.1). Next three result gives a growth and decaying estimate of all positive solutions of (1.1) under the condition (1.3), (1.4) or (1.5). 
Theorem 4.1. Let (1.3) holds. If $\left\{y_{n}\right\}$ is an eventually positive solution of (1.1), then there are positive constants $c_{1}$ and $c_{2}$ such that

$$
c_{1} \delta_{n} \leq y_{n} \leq c_{2} \gamma_{n} \text { for all large } n .
$$

Proof. In order to prove the first inequality, notice that if $\left\{y_{n}\right\}$ is a solution of type (I) or (II), it is an eventually increasing solution. Then, clearly $y_{n} \geq c \delta_{n}$ for some $c>0$ and for all large $n$. Therefore, taking into account Lemma 2.3, let us prove this inequality for a solution of type (III). Since $\left\{p_{n}\left(\Delta^{2} y_{n}\right)^{\alpha}\right\}$ is positive and increasing sequence, we get

$$
\Delta^{2} y_{n} \geq\left(\frac{c}{p_{n}}\right)^{1 / \alpha}, \quad n \geq N
$$

where $c=p_{N}\left(\Delta^{2} y_{N}\right)^{\alpha}$. Also, by Lemma 3.3 (ii), $\lim _{n \rightarrow \infty} \Delta y_{n}=\omega_{1}=0$. Then, summing (4.2) from $n$ to $m$ and letting $m \rightarrow \infty$, we obtain

$$
-\Delta y_{n} \geq c^{1 / \alpha} \sum_{s=n}^{\infty} \frac{1}{p_{s}^{1 / \alpha}}, \quad n \geq N .
$$

Summing once again obtained inequality from $n$ to $m$, letting $m \rightarrow \infty$ and using that $\lim _{n \rightarrow \infty} y_{n}=w_{0} \geq 0$, we get

$$
y_{n} \geq \omega_{0}+c^{1 / \alpha} \sum_{s=n}^{\infty} \sum_{k=s}^{\infty} \frac{1}{p_{k}^{1 / \alpha}} \geq c^{1 / \alpha} \delta_{n}, \quad n \geq N .
$$

Next, let us prove the second inequality. We consider two cases, either $\left\{y_{n}\right\}$ is a solution of type (II) i.e., $\Delta^{2} y_{n}<0$ for all large $n$ or it is a solution of type (I), or (III), i.e., $\Delta^{2} y_{n}>0$ for all large $n$. In the first case, we have $\Delta y_{n} \leq \Delta y_{N}=\lambda_{1}$, for all $n \geq N \geq n_{0}$ and summing from $N$ to $n-1$, we get $y_{n} \leq y_{N}+\Delta y_{N}(n-N), n \geq N$, from where we conclude that $\left\{y_{n} / n\right\}$ is bounded sequence. Then, $y_{n} \leq c_{2} \gamma_{n}$ for some $c_{2}>0$, since we have by (1.3) that

$$
\lim _{n \rightarrow \infty} \frac{\gamma_{n}}{n}=\lim _{n \rightarrow \infty} \frac{1}{n} \sum_{s=n_{0}}^{n-1} \sum_{k=n_{0}}^{s-1}\left(\frac{k}{p_{k}}\right)^{1 / \alpha}=\lim _{n \rightarrow \infty} \sum_{s=n_{0}}^{n-1}\left(\frac{s}{p_{s}}\right)^{1 / \alpha}=\infty .
$$

In the second case, when $\Delta^{2} y_{n}>0$ for all large $n$, by Lemma 2.1 we have that $\left\{\Delta\left(p_{n}\left(\Delta^{2} y_{n}\right)^{\alpha}\right)\right\}$ is positive and decreasing sequence, so that

$$
\Delta\left(p_{n}\left(\Delta^{2} y_{n}\right)^{\alpha}\right) \leq \Delta\left(p_{N}\left(\Delta^{2} y_{N}\right)^{\alpha}\right)=\lambda_{3}, \quad n \geq N \geq n_{0} .
$$

Summing this inequality repeatedly from $N$ to $n-1$, we obtain

$$
y_{n} \leq y_{N}+\lambda_{1}(n-N)+\sum_{s=N}^{n-1} \sum_{k=N}^{s-1} \frac{1}{p_{k}^{1 / \alpha}}\left(\lambda_{2}+\lambda_{3}(k-N)\right)^{1 / \alpha}, \quad n \geq N,
$$

where $\lambda_{1}=\Delta y_{N}, \lambda_{2}=p_{N}\left(\Delta^{2} y_{N}\right)^{\alpha}$. Now, it is easy to verify that (4.4) implies that $y_{n} \leq c_{2} \gamma_{n}$, with $c_{2}>\lambda_{3}^{1 / \alpha}>0$.

The theorem is proved. 
Therefore, under the condition (1.3), in the set of all eventually positive solutions of (1.1), a solution $\left\{y_{n}\right\}$ of type (1.8) may be regard as a "minimal" solution, while a solution $\left\{y_{n}\right\}$ of type (1.7) may be regard as a "maximal" solution.

Moreover, under the condition (1.4), in the set of all eventually positive solutions of (1.1), a solution $\left\{y_{n}\right\}$ of type (1.6) may be regard as a "minimal" solution, while a solution $\left\{y_{n}\right\}$ of type (1.9) may be regard as a "maximal" solution. Namely, we have the following theorem:

Theorem 4.2. Let (1.4) holds. If $\left\{y_{n}\right\}$ is an eventually positive solution of (1.1), then there are positive constants $c_{1}$ and $c_{2}$ such that

$$
c_{1} \alpha_{n} \leq y_{n} \leq c_{2} \beta_{n} \quad \text { for all large } n .
$$

Proof. Let $\left\{y_{n}\right\}$ be an eventually positive solution of (1.1). By Lemma 2.3 we have $\Delta y_{n}>0$ for all large $n$, so clearly there is $c_{1}>0$ such that $y_{n} \geq c_{1}$ for all large $n$.

Next, we will prove that $\left\{y_{n} / n\right\}$ is bounded sequence, so that there is $c_{2}>0$ such that $y_{n} \leq c_{2} n$ for all large $n$. We consider two cases, either $\Delta^{2} y_{n}<0$ or $\Delta^{2} y_{n}>0$, for all large $n$. In the first case, as in the proof of Theorem 4.1 we may prove that $\left\{y_{n} / n\right\}$ is bounded sequence. In the second case, when $\Delta^{2} y_{n}>0$ for all large $n$, i.e., a solution $\left\{y_{n}\right\}$ is of type (I) and accordingly $p_{n}\left(\Delta^{2} y_{n}\right)^{\alpha}>0$ for all large $n$, as in the proof of Theorem 4.1 we get (4.3). Summing (4.3) from $N$ to $n-1$, we obtain

$$
p_{n}\left(\Delta^{2} y_{n}\right)^{\alpha} \leq \lambda_{2}+\lambda_{3}(n-N), \quad n \geq N,
$$

or

$$
\Delta^{2} y_{n} \leq \lambda\left(\frac{n}{p_{n}}\right)^{1 / \alpha}, \quad n \geq N,
$$

where $\lambda=\left(\lambda_{2}+\lambda_{3}\right)^{1 / \alpha}>0$. Summing previous inequality from $N$ to $n-1$ and using the condition (1.4), we conclude that $\left\{\Delta y_{n}\right\}$ is bounded sequence. Accordingly, there is some $c_{2}>0$ such that $\Delta y_{n} \leq c_{2}$ for $n \geq N_{1} \geq N$, or we get that $y_{n} \leq y_{N_{1}}+c_{2}\left(n-N_{1}\right)$ for $n \geq N_{1}$. Therefore, we have that $\left\{y_{n} / n\right\}$ is bounded sequence.

The theorem is proved.

Under the condition (1.5), a solution $\left\{y_{n}\right\}$ of type (1.8) is a "minimal" solution in the set of all eventually positive solutions of (1.1), and a solution $\left\{y_{n}\right\}$ of type (1.9) is a "maximal" solution in the set of all eventually positive solutions of (1.1).

Theorem 4.3. Let (1.5) holds. If $\left\{y_{n}\right\}$ is an eventually positive solution of (1.1), then there are positive constants $c_{1}$ and $c_{2}$ such that

$$
c_{1} \delta_{n} \leq y_{n} \leq c_{2} \beta_{n} \quad \text { for all large } n .
$$

Proof. The first inequality may be proved as in the proof of Theorem 4.1. In order to prove the second inequality, we will again prove that $\left\{y_{n} / n\right\}$ is bounded sequence. If $\left\{y_{n}\right\}$ is a solution of type (II) or (IV), then $\Delta^{2} y_{n}<0$ for all large $n$, so that as in the proof of Theorem 4.1 we may prove that $\left\{y_{n} / n\right\}$ is bounded sequence. If $\left\{y_{n}\right\}$ is a solution of type (I), i.e., $\Delta\left(p_{n}\left(\Delta^{2} y_{n}\right)^{\alpha}\right)>0$ and $\Delta^{2} y_{n}>0$ for all large $n$, as in the proof of Theorem 4.2 we may prove that $\left\{y_{n} / n\right\}$ is bounded sequence. Finally, if $\left\{y_{n}\right\}$ is a solution of type (III), it is obviously bounded sequence and accordingly, we conclude that $\left\{y_{n} / n\right\}$ is bounded sequence.

The theorem is proved. 
5. Existence of positive solutions. In this section we give necessary and sufficient conditions for the existence of specific kinds of positive solutions.

5.1. Existence of positive solutions under the condition (1.3). Necessary and sufficient conditions for the existence of positive solutions of (1.1) satisfying (1.7) or (1.8) are given in the following two theorems.

Theorem 5.1. Suppose that (1.3) holds. Equation (1.1) has a positive solution of type (1.7) if and only if

$$
\sum_{s=n_{0}}^{\infty} q_{s} \gamma_{s+3}^{\beta}<\infty
$$

Theorem 5.2. Suppose that (1.3) holds. Equation (1.1) has a positive solution of type (1.8) if and only if

$$
\sum_{s=n_{0}}^{\infty} s q_{s} \delta_{s+3}^{\beta}<\infty
$$

The statement and the proof of Theorem 5.1 is the same as of Theorem B (Theorem 1 in [13]). Consequently, we here prove only Theorem 5.2.

Proof of Theorem 5.2. Necessity. Let $\left\{y_{n}\right\}$ be a positive solution of (1.1) of type (1.8). Then there is $N \geq n_{0}$

$$
\frac{c}{2} \delta_{n} \leq y_{n} \leq c \delta_{n}, \quad n \geq N
$$

Then, by Lemma 3.4 (ii) the solution $\left\{y_{n}\right\}$ is of the type (III), so that by Lemma 3.3 (ii) $\lim _{n \rightarrow \infty} \Delta y_{n}=0$. Moreover, $\left\{p_{n}\left(\Delta^{2} y_{n}\right)^{\alpha}\right\}$ is increasing, so we find that

$$
-\Delta y_{n}=\sum_{s=n}^{\infty} \Delta^{2} y_{s}=\sum_{s=n}^{\infty} \frac{p_{s}^{1 / \alpha} \Delta^{2} y_{s}}{p_{s}^{1 / \alpha}} \geq p_{n}^{1 / \alpha} \Delta^{2} y_{n} \sum_{s=n}^{\infty} \frac{1}{p_{s}^{1 / \alpha}}, \quad n \geq N
$$

Summing this inequality from $n$ to $m$, letting $m \rightarrow \infty$ and using that $y_{n} \rightarrow w_{0} \in[0, \infty)$, as $n \rightarrow \infty$, we obtain

$$
y_{n} \geq \sum_{s=n}^{\infty} p_{s}^{1 / \alpha} \Delta^{2} y_{s} \sum_{k=s}^{\infty} \frac{1}{p_{k}^{1 / \alpha}} \geq p_{n}^{1 / \alpha} \Delta^{2} y_{n} \delta_{n}, \quad n \geq N
$$

Accordingly,

$$
p_{n}\left(\Delta^{2} y_{n}\right)^{\alpha} \leq\left(\frac{y_{n}}{\delta_{n}}\right)^{\alpha}, \quad n \geq N
$$

which combined with (5.3) implies that $\left\{p_{n}\left(\Delta^{2} y_{n}\right)^{\alpha}\right\}$ is bounded.

Multiplying (1.1) by $n$ and summing the resulting equation from $N$ to $n-1$, we have

ISSN 1027-3190. Укр. мат. журн., 2008, m. 60, № 1 


$$
n \Delta\left(p_{n}\left(\Delta^{2} y_{n}\right)^{\alpha}\right)+\sum_{s=N}^{n-1} s q_{s} y_{s+3}^{\beta}=K+p_{n+1}\left(\Delta^{2} y_{n+1}\right)^{\alpha}, \quad n \geq N
$$

where $K$ is a constant. Since $\Delta\left(p_{n}\left(\Delta^{2} y_{n}\right)^{\alpha}\right)>0$ and $p_{n}\left(\Delta^{2} y_{n}\right)^{\alpha}$ is bounded, letting $n \rightarrow \infty$ in (5.4), we conclude that

$$
\sum_{s=N}^{\infty} s q_{s} y_{s+3}^{\beta}<\infty
$$

This, together with (5.3) implies (5.2).

Sufficiency. We assume that (5.2) holds and let $c>0$ be an arbitrary number. Then, there is $N \geq n_{0}$ such that

$$
\sum_{s=N}^{\infty}(s-N) q_{s} \delta_{s+3}^{\beta}<\frac{c^{\alpha}-(c / 2)^{\alpha}}{c^{\beta}} .
$$

Consider the Banach space $A_{N}$ of all real sequences $y=\left\{y_{n}\right\}$ with norm

$$
\|y\|=\sup _{n \geq N} \frac{\left|y_{n}\right|}{\delta_{n}}
$$

and define the set

$$
Y_{1}=\left\{\begin{array}{l|l}
y \in A_{N} & \frac{c}{2} \delta_{n} \leq y_{n} \leq c \delta_{n}
\end{array}\right\}
$$

which is clearly bounded, closed and convex subset of $A_{N}$. We will define the operator $\mathcal{G}_{1}: Y_{1} \rightarrow A_{N}$ by

$$
\left(\mathcal{G}_{1} y\right)_{n}=\sum_{s=n}^{\infty} \frac{s-n+1}{p_{s}^{1 / \alpha}}\left(c^{\alpha}-\sum_{k=s}^{\infty}(k-s+1) q_{k} y_{k+3}^{\beta}\right)^{\frac{1}{\alpha}}, \quad n \geq N
$$

The operator $\mathcal{G}_{1}$ has the following properties:

(i) $\mathcal{G}_{1}$ maps $Y_{1}$ to $Y_{1}$. For $y \in Y_{1}$, obviously

$$
\left(\mathcal{G}_{1} y\right)_{n} \leq c \delta_{n}, \quad n \geq N
$$

and using (5.5), we have

$$
\begin{aligned}
& \left(\mathcal{G}_{1} y\right)_{n} \geq \sum_{s=n}^{\infty} \frac{s-n+1}{p_{s}^{1 / \alpha}}\left(c^{\alpha}-c^{\beta} \sum_{k=s}^{\infty}(k-s+1) q_{k} \delta_{k+3}^{\beta}\right)^{\frac{1}{\alpha}} \geq \\
& \geq\left(c^{\alpha}-c^{\beta} \frac{c^{\alpha}-(c / 2)^{\alpha}}{c^{\beta}}\right)^{\frac{1}{\alpha}} \sum_{s=n}^{\infty} \frac{s-n+1}{p_{s}^{1 / \alpha}}=\frac{c}{2} \delta_{n}, \quad n \geq N .
\end{aligned}
$$

Therefore, $\mathcal{G}_{1} y \in Y_{1}$ for all $y \in Y_{1}$, i.e., $\mathcal{G}_{1}\left(Y_{1}\right) \subset Y_{1}$.

(ii) $\mathcal{G}_{1}$ is continuous on $Y_{1}$. Let $\varepsilon>0$ and let $\left\{y^{(m)}=\left(y_{1}^{(m)}, y_{2}^{(m)}, \ldots\right)\right\}$ be a sequence in $Y_{1}$, such that $\lim _{m \rightarrow \infty}\left\|y^{(m)}-y\right\|=0$. Since $Y_{1}$ is closed, $y \in Y_{1}$. We can choose $M \geq N$ so large that 


$$
\sum_{s=M}^{\infty}(s-M) q_{s} \delta_{s+3}^{\beta}<\varepsilon
$$

For all $m \in \mathbb{N}, n>N$ we have that

$$
\begin{gathered}
\left|\left(\mathcal{G}_{1} y^{(m)}\right)_{n}-\left(\mathcal{G}_{1} y\right)_{n}\right| \leq \sum_{s=n}^{\infty}(s-n+1) \frac{1}{p_{s}^{1 / \alpha}} \times \\
\times\left|\left(c^{\alpha}-\sum_{k=s}^{\infty}(k-s) q_{k}\left(y_{k+3}^{(m)}\right)^{\beta}\right)^{\frac{1}{\alpha}}-\left(c^{\alpha}-\sum_{k=s}^{\infty}(k-s) q_{k} y_{k+3}^{\beta}\right)^{\frac{1}{\alpha}}\right|= \\
=\sum_{s=n}^{\infty} \frac{s-n+1}{p_{s}^{1 / \alpha}}\left|F_{s}^{(m)}-F_{s}\right|,
\end{gathered}
$$

where

$$
F_{s}^{(m)}=\left(c^{\alpha}-\sum_{k=s}^{\infty}(k-s) q_{k}\left(y_{k+3}^{(m)}\right)^{\beta}\right)^{\frac{1}{\alpha}}, \quad F_{s}=\left(c^{\alpha}-\sum_{k=s}^{\infty}(k-s) q_{k} y_{k+3}^{\beta}\right)^{\frac{1}{\alpha}} .
$$

Since, using (5.7), we have for all large $m \in \mathbb{N}$ and all $s \geq N$ that

$$
\begin{gathered}
\quad\left|\sum_{k=s}^{\infty}(k-s) q_{k}\left(y_{k+3}^{(m)}\right)-\sum_{k=s}^{\infty}(k-s) q_{k} y_{k+3}^{\beta}\right| \leq \\
\leq\left|\sum_{k=s}^{M}(k-s) q_{k}\left(y_{k+3}^{(m)}\right)^{\beta}-\sum_{k=s}^{M}(k-s) q_{k} y_{k+3}^{\beta}\right|+ \\
+\left|\sum_{k=M}^{\infty}(k-M) q_{k}\left(y_{k+3}^{(m)}\right)^{\beta}\right|+\left|\sum_{k=M}^{\infty}(k-M) q_{k} y_{k+3}^{\beta}\right| \leq \\
\leq\left|\sum_{k=s}^{M}(k-s) q_{k}\left(y_{k+3}^{(m)}\right)^{\beta}-\sum_{k=s}^{M}(k-s) q_{k} y_{k+3}^{\beta}\right|+ \\
+2 c^{\beta} \sum_{k=M}^{\infty}(k-M) q_{k} \delta_{k+3}^{\beta}<3 \varepsilon .
\end{gathered}
$$

Therefore, $\lim _{m \rightarrow \infty}\left|F_{s}^{(m)}-F_{s}\right|=0$, for each $s \geq N$. Now, from (5.8) we get that for all large $m \in \mathbb{N}$

$$
\left|\left(\mathcal{G}_{1} y^{(m)}\right)_{n}-\left(\mathcal{G}_{1} y\right)_{n}\right|<\varepsilon \sum_{s=n}^{\infty} \frac{s-n+1}{p_{s}^{1 / \alpha}}=\varepsilon \delta_{n}, \quad n>N
$$

which shows that $\left\|\mathcal{G}_{1} y^{(m)}-\mathcal{G}_{1} y\right\| \rightarrow 0$ as $m \rightarrow \infty$, i.e., that $\mathcal{G}_{1}$ is continuous on $Y_{1}$.

(iii) $\mathcal{G}_{1}\left(Y_{1}\right)$ is uniformly Cauchy. To see this, we have to show that for any given $\varepsilon>0$, there exists an integer $M_{1}$ such that for $m>n \geq M_{1}$ 


$$
\left|\frac{\left(\mathcal{G}_{1} y\right)_{m}}{\delta_{m}}-\frac{\left(\mathcal{G}_{1} y\right)_{n}}{\delta_{n}}\right|<\varepsilon
$$

for any $y \in Y_{1}$. Indeed, by (5.6), for some $y \in Y_{1}$ and $m>n \geq N$, we have

$$
\begin{aligned}
& \left|\frac{\left(\mathcal{G}_{1} y\right)_{m}}{\delta_{m}}-\frac{\left(\mathcal{G}_{1} y\right)_{n}}{\delta_{n}}\right|= \\
& =\mid \frac{1}{\delta_{m}} \sum_{s=m}^{\infty}(s-m+1) \frac{1}{p_{s}^{1 / \alpha}}\left(c^{\alpha}-\sum_{k=s}^{\infty}(k-s) q_{k} y_{k+3}^{\beta}\right)^{\frac{1}{\alpha}}- \\
& -\frac{1}{\delta_{n}} \sum_{s=n}^{\infty}(s-n+1) \frac{1}{p_{s}^{1 / \alpha}}\left(c^{\alpha}-\sum_{k=s}^{\infty}(k-s) q_{k} y_{k+3}^{\beta}\right)^{\frac{1}{\alpha}} \mid \leq \\
& \leq \frac{1}{\delta_{n}} \mid \sum_{s=n}^{\infty}(s-n+1) \frac{1}{p_{s}^{1 / \alpha}}\left(c^{\alpha}-\sum_{k=s}^{\infty}(k-s) q_{k} y_{k+3}^{\beta}\right)^{\frac{1}{\alpha}}- \\
& -\sum_{s=m}^{\infty}(s-m+1) \frac{1}{p_{s}^{1 / \alpha}}\left(c^{\alpha}-\sum_{k=s}^{\infty}(k-s) q_{k} y_{k+3}^{\beta}\right)^{\frac{1}{\alpha}} \mid+ \\
& +\left|\frac{1}{\delta_{m}}-\frac{1}{\delta_{n}}\right| \sum_{s=m}^{\infty}(s-m+1) \frac{1}{p_{s}^{1 / \alpha}}\left(c^{\alpha}-\sum_{k=s}^{\infty}(k-s) q_{k} y_{k+3}^{\beta}\right)^{\frac{1}{\alpha}} \leq \\
& \leq \frac{1}{\delta_{n}} \sum_{s=n}^{m-1}(s-n+1) \frac{1}{p_{s}^{1 / \alpha}}\left(c^{\alpha}-\sum_{k=s}^{\infty}(k-s) q_{k} y_{k+3}^{\beta}\right)^{\frac{1}{\alpha}}+ \\
& +\frac{2}{\delta_{m}} \sum_{s=m}^{\infty}(s-m+1) \frac{1}{p_{s}^{1 / \alpha}}\left(c^{\alpha}-\sum_{k=s}^{\infty}(k-s) q_{k} y_{k+3}^{\beta}\right)^{\frac{1}{\alpha}} \leq \\
& \leq \frac{1}{\delta_{n}} \sum_{s=n}^{\infty}(s-n+1) \frac{1}{p_{s}^{1 / \alpha}}\left(c^{\alpha}-\sum_{k=s}^{\infty}(k-s) q_{k} y_{k+3}^{\beta}\right)^{\frac{1}{\alpha}}+ \\
& +\frac{2}{\delta_{m}} \sum_{s=m}^{\infty}(s-m+1) \frac{1}{p_{s}^{1 / \alpha}}\left(c^{\alpha}-\sum_{k=s}^{\infty}(k-s) q_{k} y_{k+3}^{\beta}\right)^{\frac{1}{\alpha}} \leq \\
& \leq \frac{1}{\delta_{n}} \delta_{n}\left(c^{\alpha}-\sum_{k=N}^{\infty}(k-N) q_{k} y_{k+3}^{\beta}\right)^{\frac{1}{\alpha}}+ \\
& +\frac{2}{\delta_{m}} \delta_{m}\left(c^{\alpha}-\sum_{k=N}^{\infty}(k-N) q_{k} y_{k+3}^{\beta}\right)^{\frac{1}{\alpha}} \leq \\
& \leq 3\left(c^{\alpha}+\sum_{k=N}^{\infty}(k-N) q_{k} y_{k+3}^{\beta}\right)^{\frac{1}{\alpha}} \leq 3\left(c^{\alpha}+c^{\beta} \sum_{k=N}^{\infty}(k-N) q_{k} \delta_{k+3}^{\beta}\right)^{\frac{1}{\alpha}} .
\end{aligned}
$$


Using the condition (5.2), it is clear that $\mathcal{G}_{1}\left(Y_{1}\right)$ is uniformly Cauchy.

Accordingly, by Lemma 3.1, we conclude that there exists an $\widetilde{y} \in Y_{1}$ such that $\mathcal{G}_{1} \widetilde{y}=\widetilde{y}$. It is easy to check that $\widetilde{y}=\left\{\widetilde{y}_{n}\right\}$ is a positive solution of (1.1). Moreover, clearly

$$
\lim _{n \rightarrow \infty} \frac{\widetilde{y}_{n}}{\delta_{n}} \leq c
$$

and using (5.6) we have that

$$
\begin{gathered}
\lim _{n \rightarrow \infty} \frac{\widetilde{y}_{n}}{\delta_{n}} \geq \lim _{n \rightarrow \infty} \frac{\sum_{s=n}^{\infty} \frac{s-n+1}{p_{s}^{1 / \alpha}}\left(c^{\alpha}-\sum_{k=n}^{\infty}(k-n) q_{k} \widetilde{y}_{k+3}^{\beta}\right)^{\frac{1}{\alpha}}}{\sum_{s=n}^{\infty}(s-n+1) \frac{1}{p_{s}^{1 / \alpha}}}= \\
=\lim _{n \rightarrow \infty}\left(c^{\alpha}-\sum_{k=n}^{\infty}(k-n) q_{k} \widetilde{y}_{k+3}^{\beta}\right)^{\frac{1}{\alpha}}=c .
\end{gathered}
$$

Therefore, we have

$$
\lim _{n \rightarrow \infty} \frac{\widetilde{y}_{n}}{\delta_{n}}=c
$$

The theorem is proved.

5.2. Existence of positive solutions under the condition (1.4). Now, we present necessary and sufficient condition for the existence of positive solutions satisfying (1.6).

Theorem 5.3. Suppose that (1.4) holds. Equation (1.1) has a positive solution which satisfies (1.6) if and only if

$$
\sum_{n=n_{0}}^{\infty} \frac{n}{p_{n}^{1 / \alpha}}\left(\sum_{s=n}^{\infty}(s-n) q_{s}\right)^{\frac{1}{\alpha}}<\infty .
$$

The statement of Theorem 5.3 is the same as of Theorem A (Theorem 2 in [13]), except that instead of the assumption (1.2) it is assumed that (1.4) holds. If (1.4) holds and $\left\{y_{n}\right\}$ is a positive solution which satisfies (1.6), by Lemma 3.4 (iii) the solution $\left\{y_{n}\right\}$ is of type (II). Therefore, the proof of necessity part of Theorem 5.3 and Theorem A is the same. Moreover, as in the proof of Theorem A we may prove that the condition (5.9) is sufficient for the existence of solution of type (1.6). Consequently, the proof of Theorem 5.3 is essentially given in [13] (Theorem 2).

Now, we turn to the existence of positive solutions of type (1.9). We will consider the Banach space $B_{N}$ of all real sequences $y=\left\{y_{n}\right\}$ with norm

$$
\|y\|=\sup _{n \geq N} \frac{\left|y_{n}\right|}{n}
$$

and define sets

$$
H_{1}=\left\{y \in B_{N} \mid \frac{c}{2}(n-N) \leq y_{n} \leq c(n-N), n \geq N\right\},
$$

and 


$$
H_{2}=\left\{y \in B_{N} \mid c(n-N) \leq y_{n} \leq 2 c(n-N), n \geq N\right\},
$$

which are both clearly bounded, closed and convex subsets of $B_{N}$.

Theorem 5.4. Suppose that (1.4) holds. Equation (1.1) has a positive solution of type (I) which satisfies (1.9) if and only if

$$
\sum_{n=n_{0}}^{\infty}(n+3)^{\beta} q_{n}<\infty
$$

Proof. Necessity. Let $\left\{y_{n}\right\}$ be a positive solution of (1.1) of type (I) which satisfies (1.9). Then there is $N \geq n_{0}$ such that (I) holds for all $n \geq N$ and

$$
c n \leq y_{n} \leq 2 c n, \quad n \geq N .
$$

Summing (1.1) from $n$ to $k-1$ we get

$$
\Delta\left(p_{n}\left(\Delta^{2} y_{n}\right)^{\alpha}\right)=\Delta\left(p_{k}\left(\Delta^{2} y_{k}\right)^{\alpha}\right)+\sum_{i=n}^{k-1} q_{i} y_{i+3}^{\beta}, \quad k>n \leq N .
$$

Since $\left\{\Delta\left(p_{k}\left(\Delta^{2} y_{k}\right)^{\alpha}\right)\right\}$ is positive and decreasing sequence, it tends to a finite limit $w_{3} \geq 0$ as $k \rightarrow \infty$. Then, letting $k \rightarrow \infty$ in (5.12) we have

$$
\Delta\left(p_{n}\left(\Delta^{2} y_{n}\right)^{\alpha}\right)=w_{3}+\sum_{i=n}^{\infty} q_{i} y_{i+3}^{\beta} \geq \sum_{i=n}^{\infty} q_{i} y_{i+3}^{\beta}, \quad n \geq N .
$$

Then, using (5.11) from (5.13) we get

$$
\Delta\left(p_{N}\left(\Delta^{2} y_{N}\right)^{\alpha}\right) \geq c^{\beta} \sum_{k=N}^{\infty}(k+3)^{\beta} q_{k},
$$

which proves that (5.10) holds.

Sufficiency. Let $c>0$ be an arbitrary number. We assume that (1.4) and (5.10) hold. Then, there is $N \geq n_{0}$ such that

$$
Q=\sum_{k=N}^{\infty}(k+3)^{\beta} q_{k}, \quad 2 Q^{\frac{1}{\alpha}} \sum_{k=N}^{\infty}\left(\frac{k-N}{p_{k}}\right)^{\frac{1}{\alpha}} \leq c^{1-\frac{\beta}{\alpha}} .
$$

We will define the operator $\mathcal{H}_{1}: H_{1} \rightarrow B_{N}$ by

$$
\left(\mathcal{H}_{1} y\right)_{n}=c(n-N)-\sum_{k=N}^{n-1} \sum_{j=k}^{\infty} \frac{1}{p_{j}^{1 / \alpha}}\left(\sum_{i=N}^{j-1} \sum_{s=i}^{\infty} q_{s} y_{s+3}^{\beta}\right)^{\frac{1}{\alpha}}, \quad n \geq N .
$$

For $y \in H_{1}$, using (5.14), we have

$$
c(n-N) \geq\left(\mathcal{H}_{1} y\right)_{n} \geq c(n-N)-c^{\frac{\beta}{\alpha}} \sum_{k=N}^{n-1} \sum_{j=k}^{\infty} \frac{1}{p_{j}^{1 / \alpha}}\left(\sum_{i=N}^{j-1} \sum_{s=i}^{\infty}(s+3)^{\beta} q_{s}\right)^{\frac{1}{\alpha}} \geq
$$




$$
\begin{gathered}
\geq c(n-N)-c^{\frac{\beta}{\alpha}} Q^{\frac{1}{\alpha}} \sum_{k=N}^{n-1} \sum_{j=k}^{\infty}\left(\frac{j-N}{p_{j}}\right)^{\frac{1}{\alpha}} \geq \\
\geq\left(c-\frac{1}{2} c^{\frac{\beta}{\alpha}} c^{1-\frac{\beta}{\alpha}}\right)(n-N)=\frac{c}{2}(n-N), \quad n \geq N .
\end{gathered}
$$

Therefore, $\mathcal{H}_{1} y \in H_{1}$ for all $y \in H_{1}$, i.e., $\mathcal{H}_{1}$ maps $H_{1}$ to $H_{1}$. Moreover, in the similar way as in the proof of Theorem 5.2, we may show that the operator $\mathcal{H}_{1}$ is continuous on $H_{1}$ and $\mathcal{H}_{1}\left(H_{1}\right)$ is uniformly Cauchy. In view of Lemma 3.1, we see that there exists an $\widehat{y} \in H_{1}$ such that $\mathcal{H}_{1} \widehat{y}=\widehat{y}$. It is easy to see that $\widehat{y}=\left\{\widehat{y}_{n}\right\}$ is a positive solution of (1.1) of type (I). Furthermore, from (5.15), using the condition (1.4), we have that

$$
\begin{gathered}
c \geq \lim _{n \rightarrow \infty} \frac{\widehat{y}_{n}}{n}=\lim _{n \rightarrow \infty} \frac{\left(\mathcal{H}_{1} \widehat{y}\right)_{n}}{n} \geq c-c^{\beta / \alpha} Q^{1 / \alpha} \lim _{n \rightarrow \infty} \frac{1}{n} \sum_{k=N}^{n-1} \sum_{j=k}^{\infty}\left(\frac{j-N}{p_{j}}\right)^{\frac{1}{\alpha}}= \\
=c-c^{\beta / \alpha} Q^{1 / \alpha} \lim _{n \rightarrow \infty} \sum_{j=n}^{\infty}\left(\frac{j-N}{p_{j}}\right)^{\frac{1}{\alpha}}=c,
\end{gathered}
$$

which shows that the solution $\widehat{y}$ is of type (1.9).

The theorem is proved.

Theorem 5.5. Suppose that (1.4) holds. Equation (1.1) has a positive solution of type (II) which satisfies (1.9) if and only if

$$
\sum_{n=n_{0}}^{\infty} n(n+3)^{\beta} q_{n}<\infty
$$

Proof. Necessity. Let $\left\{y_{n}\right\}$ be a positive solution of (1.1) of type (II) which satisfies (1.9), so that there is $N \geq n_{0}$ such that (II) and (5.11) hold for all $n \geq N$. Then, since $\left\{\Delta\left(p_{n}\left(\Delta^{2} y_{n}\right)^{\alpha}\right)\right\}$ is again positive and decreasing sequence, as in the proof of Theorem 5.4 we get (5.13) for all $n \geq N$. Moreover, by Lemma 3.3 (i), $\lim _{n \rightarrow \infty} \Delta\left(p_{n}\left(\Delta^{2} y_{n}\right)^{\alpha}\right)=w_{3}=0$ and (5.13) becomes

$$
\Delta\left(p_{n}\left(\Delta^{2} y_{n}\right)^{\alpha}\right)=\sum_{k=n}^{\infty} q_{k} y_{k+3}^{\beta}, \quad n \geq N .
$$

Summing this inequality from $N$ to $n-1$ and using (5.11) we get

$$
p_{n}\left(\Delta^{2} y_{n}\right)^{\alpha}=\xi_{2}+\sum_{k=N}^{n-1} \sum_{i=k}^{\infty} q_{i} y_{i+3}^{\beta} \geq \xi_{2}+c^{\beta} \sum_{k=N}^{n-1} \sum_{i=k}^{\infty}(i+3)^{\beta} q_{i}, \quad n \geq N,
$$

where $\xi_{2}=p_{N}\left(\Delta^{2} y_{N}\right)^{\alpha}<0$. Since $\left\{p_{n}\left(\Delta^{2} y_{n}\right)^{\alpha}\right\}$ is negative and increasing sequence, there exist $\lim _{n \rightarrow \infty} p_{n}\left(\Delta^{2} y_{n}\right)^{\alpha}=w_{2} \leq 0$. Accordingly, letting $n \rightarrow \infty$ in (5.17) we have

$$
\sum_{k=N}^{\infty}(k-N+1)(k+3)^{\beta} q_{k}<\infty
$$

which proves that (5.16) is satisfied. 
Sufficiency. We assume that (5.16) holds. Moreover, the condition (1.4) implies that

$$
\sum_{n=n_{0}}^{\infty} \frac{1}{p_{n}^{1 / \alpha}}<\infty
$$

Therefore, for an arbitrary positive constant $c$ there is $N \geq n_{0}$ such that

$$
\sum_{k=N}^{\infty} \frac{1}{p_{k}^{1 / \alpha}} \leq 1, \quad 2^{\beta} \sum_{k=N}^{\infty}(k-N)(k+3)^{\beta} q_{k} \leq c^{\alpha-\beta} .
$$

A solution of (1.1) satisfying (II) and (1.9) may be obtained as a fixed point of the operator $\mathcal{H}_{2}: H_{2} \rightarrow B_{N}$ defined by

$$
\left(\mathcal{H}_{2} y\right)_{n}=c(n-N)+\sum_{k=N}^{n-1} \sum_{j=k}^{\infty} \frac{1}{p_{j}^{1 / \alpha}}\left(c^{\alpha}-\sum_{i=N}^{j-1} \sum_{s=i}^{\infty} q_{s} y_{s+3}^{\beta}\right)^{\frac{1}{\alpha}}, \quad n \geq N
$$

The operator $\mathcal{H}_{2}$ satisfies the assumptions of Lemma 3.1. Indeed, for all $y \in H_{2}$, using (5.18), we have

$$
\begin{gathered}
\left(\mathcal{H}_{2} y\right)_{n} \geq c(n-N)+\sum_{k=N}^{n-1} \sum_{j=k}^{\infty} \frac{1}{p_{j}^{1 / \alpha}}\left(c^{\alpha}-(2 c)^{\beta} \sum_{i=N}^{j-1} \sum_{s=i}^{\infty}(s+3)^{\beta} q_{s}\right)^{\frac{1}{\alpha}} \geq \\
\geq c(n-N)+\sum_{k=N}^{n-1} \sum_{j=k}^{\infty} \frac{1}{p_{j}^{1 / \alpha}}\left(c^{\alpha}-(2 c)^{\beta} \sum_{i=N}^{\infty} \sum_{s=i}^{\infty}(s+3)^{\beta} q_{s}\right)^{\frac{1}{\alpha}} \geq \\
\geq c(n-N)+\sum_{k=N}^{n-1} \sum_{j=k}^{\infty} \frac{1}{p_{j}^{1 / \alpha}}\left(c^{\alpha}-(2 c)^{\beta} \sum_{i=N}^{\infty}(i-N)(i+3)^{\beta} q_{i}\right)^{\frac{1}{\alpha}} \geq \\
\geq c(n-N)+\sum_{k=N}^{n-1} \sum_{j=k}^{\infty} \frac{1}{p_{j}^{1 / \alpha}}\left(c^{\alpha}-c^{\beta} c^{\alpha-\beta}\right)^{\frac{1}{\alpha}}=c(n-N), \quad n \geq N,
\end{gathered}
$$

and

$$
\left(\mathcal{H}_{2} y\right)_{n} \leq \sum_{k=N}^{n-1}\left(c+c \sum_{j=k}^{\infty} \frac{1}{p_{j}^{1 / \alpha}}\right) \leq 2 c(n-N), \quad n \geq N
$$

Therefore, $\mathcal{H}_{2}$ maps $H_{2}$ to $H_{2}$. We may verify that the operator $\mathcal{H}_{2}$ is continuous on $\mathrm{H}_{2}$ as well as that $\mathcal{H}_{2}\left(H_{2}\right)$ is uniformly Cauchy. Therefore, by Lemma 3.1 we conclude that there exists an $\widehat{y} \in H_{2}$ such that $\mathcal{H}_{2} \widehat{y}=\widehat{y}$. It is easy to see that $\widehat{y}=\left\{\widehat{y}_{n}\right\}$ is a positive solution of (1.1) of type (II). Furthermore, by application of Stolz's theorem, we have that

$$
c \leq \lim _{n \rightarrow \infty} \frac{\widehat{y}_{n}}{n}=\lim _{n \rightarrow \infty} \frac{\left(\mathcal{H}_{2} \widehat{y}\right)_{n}}{n} \leq c+c \lim _{n \rightarrow \infty} \sum_{j=n}^{\infty} \frac{1}{p_{j}^{1 / \alpha}}=c
$$

which shows that the solution $\widehat{y}$ is of type (1.9).

The theorem is proved. 
Theorem 5.6. Equation (1.1) has a positive solution of type (IV) satisfying (1.9) if and only if

$$
\sum_{n=n_{0}}^{\infty} \frac{1}{p_{n}^{1 / \alpha}}\left(\sum_{k=n_{0}}^{n-1}(n-k-1)(k+3)^{\beta} q_{k}\right)^{\frac{1}{\alpha}}<\infty
$$

Proof. Necessity. Let $\left\{y_{n}\right\}$ be a positive solution of (1.1) of type (IV) which satisfies (1.9). Then there is $N \geq n_{0}$ such that (IV) and (5.11) hold for all $n \geq N$. Summing (1.1) twice from $N$ to $n-1$ we have

$$
p_{n}\left(\Delta^{2} y_{n}\right)^{\alpha}=\xi_{2}+\xi_{3}(n-N)-\sum_{k=N}^{n-1} \sum_{i=N}^{k-1} q_{i} y_{i+3}^{\beta}, \quad n \geq N,
$$

where $\xi_{2}=p_{N}\left(\Delta^{2} y_{N}\right)^{\alpha}<0$ and $\xi_{3}=\Delta\left(p_{N}\left(\Delta^{2} y_{N}\right)^{\alpha}\right)<0$. Accordingly,

$$
p_{n}\left(\Delta^{2} y_{n}\right)^{\alpha} \leq-\sum_{k=N}^{n-1}(n-k-1) q_{k} y_{k+3}^{\beta}, \quad n \geq N
$$

implying that

$$
-\Delta^{2} y_{n} \geq \frac{1}{p_{n}^{1 / \alpha}}\left(\sum_{k=N}^{n-1}(n-k-1) q_{k} y_{k+3}^{\beta}\right)^{1 / \alpha}, \quad n \geq N .
$$

For the solution $\left\{y_{n}\right\}$ of type (IV), $\left\{\Delta y_{n}\right\}$ is positive and decreasing sequence, so there exits $\lim _{n \rightarrow \infty} \Delta y_{n}=w_{1}, 0 \leq w_{1}<\infty$. Therefore, summing the previous inequality from $N$ to $r-1$, letting $r \rightarrow \infty$ and using (5.11) we get

$$
\Delta y_{N} \geq c^{\beta} \sum_{k=N}^{\infty} \frac{1}{p_{k}^{1 / \alpha}}\left(\sum_{i=N}^{k-1}(k-i-1)(i+3)^{\beta} q_{i}\right)^{1 / \alpha} .
$$

Accordingly, we conclude that (5.20) is satisfied.

Sufficiency. We assume that (5.20) holds and let $c>0$ be an arbitrary number. Then, there is $N \geq n_{0}$ such that

$$
2^{\frac{\beta}{\alpha}} \sum_{k=N}^{\infty} \frac{1}{p_{k}^{1 / \alpha}}\left(\sum_{i=N}^{k-1}(k-i-1)(i+3)^{\beta} q_{i}\right)^{\frac{1}{\alpha}} \leq c^{1-\frac{\beta}{\alpha}} .
$$

We will define the operator $\mathcal{H}_{3}: H_{2} \rightarrow B_{N}$ by

$$
\left(\mathcal{H}_{3} y\right)_{n}=c(n-N)+\sum_{k=N}^{n-1} \sum_{i=k}^{\infty} \frac{1}{p_{i}^{1 / \alpha}}\left(\sum_{j=N}^{i-1}(i-j-1) q_{j} y_{j+3}^{\beta}\right)^{\frac{1}{\alpha}}, \quad n \geq N .
$$

By Lemma 3.1, we may conclude that there exists an $\widehat{y} \in H_{2}$ such that $\mathcal{H}_{3} \widehat{y}=\widehat{y}$. The operator $\mathcal{H}_{3}$ satisfies the assumptions of Lemma 3.1, since $\mathcal{H}_{3}$ is the continuous operator on $H_{2}, \mathcal{H}_{3}\left(H_{2}\right)$ is uniformly Cauchy and for all $y \in H_{2}$, using (5.22), we have 


$$
\begin{gathered}
c(n-N) \leq\left(\mathcal{H}_{3} y\right)_{n} \leq \\
\leq c(n-N)+(2 c)^{\frac{\beta}{\alpha}} \sum_{k=N}^{n-1} \sum_{i=k}^{\infty} \frac{1}{p_{i}^{1 / \alpha}}\left(\sum_{j=N}^{i-1}(i-j-1)(j+3)^{\beta} q_{j}\right)^{\frac{1}{\alpha}} \leq \\
\leq c(n-N)+c^{\frac{\beta}{\alpha}} c^{1-\frac{\beta}{\alpha}}(n-N)=2 c(n-N), \quad n \geq N,
\end{gathered}
$$

so that $\mathcal{H}_{3}$ maps $H_{2}$ to $H_{2}$.

It is easy to see that $\widehat{y}=\left\{\widehat{y}_{n}\right\}$ is a positive solution of (1.1) of type (IV) and by Stolz's theorem, taking into account the assumption (5.20), we have that

$$
\lim _{n \rightarrow \infty} \frac{\widehat{y}_{n}}{n}=\lim _{n \rightarrow \infty} \frac{\left(\mathcal{H}_{3} \widehat{y}\right)_{n}}{n}=c
$$

which shows that the solution $\widehat{y}$ is of type (1.9).

The theorem is proved.

Nothing that under the condition (1.4), we have that

$$
(5.16) \Rightarrow(5.10) \Rightarrow(5.20)
$$

we have the following result on the existence of the positive solution of type (1.9), under the assumptio (1.4).

Theorem 5.7. Suppose that (1.4) holds. Equation (1.1) has a positive solution which satisfies (1.9) if and only if (5.20) holds.

5.3. Existence of positive solutions under the condition (1.5). If we suppose that (1.5) holds and $\left\{y_{n}\right\}$ is a positive solution which satisfies (1.8), by Lemma 3.4 (ii) the solution $\left\{y_{n}\right\}$ is of type (III). Therefore, under the condition (1.5), as in the proof of Theorem 5.2 we may prove that the condition (5.2) is necessary for the existence of positive solution of type (1.8). On the other hand, in the sufficiently part of the proof of Theorems 5.2, only the first part of the condition (1.3) has been used. Therefore, the statement of Theorem 5.2 remains to hold if the condition (1.5) is assumed to hold and we have the following result on the existence of solution of type (1.8):

Theorem 5.8. Suppose that (1.5) holds. The condition (5.2) is a necessary and sufficient condition for the equation (1.1) to have a positive solution $\left\{y_{n}\right\}$ which satisfies (1.8).

Notice that in the sufficiently part of the proof of Theorems 5.4-5.6, we used only the second part of the condition (1.4), i.e., that

$$
\sum_{n=n_{0}}^{\infty}\left(\frac{n}{p_{n}}\right)^{1 / \alpha}<\infty .
$$

Moreover, if (1.5) holds, by Lemma 2.3, (I) or (II) or (III) or (IV) holds. If $\left\{y_{n}\right\}$ is a positive solution which satisfies (1.9), it can not be of type (III), because if we suppose on the contrary that $\left\{y_{n}\right\}$ is a positive and decreasing sequence, we would have that $\lim _{n \rightarrow \infty} y_{n} / n=0$. Accordingly, if (1.5) holds we can prove in the same way Theorems $5.4-5.6$, so that we have the following results: 
Theorem 5.9. Suppose that (1.5) holds. The condition (5.20) is a necessary and sufficient condition for the equation (1.1) to have a positive solution $\left\{y_{n}\right\}$ which satisfies (1.9).

1. Agarwal R. P., Bohner M., Grace S. R., O'Regan D. Discrete oscillation theory. - New York: Hindawi Publ., 2005.

2. Agarwal R. P. Difference equations and inequalities. - New York: Marcel Dekker, 1992.

3. Agarwal R. P., Grace S. R., O'Regan D. On the oscillation of certain third-order difference equations // Adv. Difference Equat. - 2005. - 2005, № 3. - P. 345 - 367 .

4. Grace S. R., Lalli B. S. Oscillation of higher-order nonlinear difference equations // Math. Comput. Modell. - 2005. - 41. - P. $485-491$.

5. Li W. T., Agarwal R. P. Positive solutions of higher-order nonlinear delay difference equations // Comput. Math. Appl. - 2003. - 45. - P. $1203-1211$.

6. Li W. T., Cheng S. S., Zhang G. A classification scheme for nonoscillatory solutions of a higher-order neutral nonlinear difference equation // J. Austral. Math. Soc. Ser. A. - 1999. - 67. - P. $122-142$.

7. Zhang B., Sun Y. J. Classification of nonoscillatory solutions of a higher order neutral difference equation // J. Difference Equat. and Appl. - 2002. - 8. - P. 937-955.

8. Zhu Z., Wang G., Cheng S. S. A classification scheme for nonoscillatory solutions of a higher order neutral difference equation // Adv. Difference Equat. - 2006. - 2006. - P. 1-19.

9. Migda M., Schmeidel E. Asymptotic properties of fourth-order nonlinear difference equations // Math. Comput. Modell. - 2004. - 39. - P. $1203-1211$.

10. Migda M., Musielak A., Schmeidel E. On a class of fourth-order nonlinear difference equations // Adv. Difference Equat. - 2004. - 2004, № 1. - P. 23-36.

11. Liu B., Yan J. Oscillatory and asymptotic behavior of fourth-order nonlinear difference equations // Acta math. sinica. - 1997. - 13. - P. $105-115$.

12. Schmeidel E. Oscillation and nonoscillation theorems for fourth order difference equations // Rocky Mountain J. Math. - 2003. - 33. - P. 1083 - 1094.

13. Thandapani E., Selvaraj B. Oscillatory and nonoscillatory behavior of fourth order quasilinear difference equations // Far East J. Appl. Math. - 2004. - 17, № 3. - P. 287-307.

14. Zhang B. G., Cheng S. S. On a class of nonlinear difference equations // J. Difference Equat. and Appl. - 1995. - 1. - P. $391-411$.

15. Agarwal R. P., Manojlović J. V. Asymptotic behavior of nonoscillatory solutions of fourth order nonlinear difference equations // Dynam. Continuous, Discrete and Impulsive Systems (to appear).

Received 26.06.07 